\title{
Singular traveling wave solutions for Boussinesq equation with power law nonlinearity and dual dispersion
}

\author{
Shan Zheng ${ }^{1}$, Zhengyong Ouyang ${ }^{2^{*}}(1)$ and Kuilin $\mathrm{Wu}^{3}$
}

"Correspondence: oyzy1128@126.com ${ }^{2}$ Department of Mathematics, Foshan University, Foshan, P.R. China Full list of author information is available at the end of the article

\section{Springer}

\begin{abstract}
In this paper we study the Boussinesq equation with power law nonlinearity and dual dispersion which arises in fluid dynamics. A particular kind of product of distributions is introduced and applied to solve non-smooth solutions of this equation. It is proved that, under certain conditions, a distribution solution as a singular Dirac delta function exists for this model. For the first time, this kind of product of distributions is used to deal with a fourth order nonlinear partial differential equation.
\end{abstract}

Keywords: Boussinesq equation; Dual dispersion; Product of distributions; Traveling delta wave solution

\section{Introduction}

In 1872, Boussinesq [1] derived one-dimensional nonlinear water wave equation under the assumption that the horizontal velocity is constant along the water depth, and the vertical velocity is linear along the water depth, and it is called the Boussinesq equation. The classical Boussinesq equation is the momentum equation of mass conservation and incompressible inviscid fluid. It has the following properties: (1) the governing equation is expressed by water depth and velocity, which satisfies the conservation of mass and momentum in any case, so it can describe wave refraction, diffraction, and the interaction between waves and reflected waves; (2) the Boussinesq equation is weak dispersive and nonlinear, it is only suitable for the shallow water area; (3) the classical Boussinesq equation cannot be used to deal with the strong nonlinearity of wave breaking and the influence of environmental current. There are more details about advantages and disadvantages and the application of Boussinesq equation in [2-10].

However, some characteristics of the classic Boussinesq equation limit the application of the equation in wider range. In order to expand the range of water depth of the equation, many researchers came to study the modified or generalized forms of Boussinesq equation, so that it can be applied to the deep water area. For example, the velocity variation or the higher derivative term is introduced to adjust the linear dispersion performance of the equation.

(c) The Author(s) 2019. This article is distributed under the terms of the Creative Commons Attribution 4.0 International License (http://creativecommons.org/licenses/by/4.0/), which permits unrestricted use, distribution, and reproduction in any medium, provided you give appropriate credit to the original author(s) and the source, provide a link to the Creative Commons license, and indicate if changes were made. 
This paper is devoted to the study of Boussinesq equation with power law nonlinearity and dual dispersion that is investigated in fluid dynamics [11-13] as follows:

$$
u_{t t}-k^{2} u_{x x}+a\left(u^{2 n}\right)_{x x}+b_{1} u_{x x x x}+b_{2} u_{x x t t}=0
$$

where $u(x, t)$ represents the wave profile, $x$ and $t$ are spacial and temporal variables, respectively, in addition, $k, a$, and $b_{j}$ for $j=1,2$ are real-valued constants. The first term means the developing term, the first two terms form the wave operator, the term with coefficient $a$ represents the nonlinear action, where $n$ is the power law nonlinearity parameter. Then, the two terms with coefficients $b_{j}(j=1,2)$ are the dispersions, where the first one is the regular dispersion, while the second one arises as the surface tension [14].

Special solutions play an important role in the research of partial differential equations, and they can be used to describe and explain many phenomena in physics and engineering and so on. It is interesting to consider the different kinds of exact solutions of (1.1). There were some results involving exact solutions to be obtained, here we give a brief review. Equation (1.1) was studied in order to look for exact solutions in [11], there three integration tools were adopted in order to extract the soliton solutions. These methods used in [11] are the traveling wave hypothesis, ansatz method, and the semi-inverse variational principle. The shock waves and singular soliton solutions to (1.1) were obtained and the wave profiles were also displayed numerically. Besides, the connection between singular solitons and solitary waves was also established. The conserved quantities were also obtained by the aid of multiplier method in Lie symmetry. Soliton solutions of (1.1) in two forms were considered in [12]. The solitary wave ansatz was used to carry out the integration of these equations. Two of the conserved quantities were laid down. Finally, the numerical simulation was carried out for these two equations as well. In [13], from the view of integrability as well, the Boussinesq equation with power law nonlinearity and dual dispersion (1.1) was studied, three additional algorithms were used to search for solutions; as a result, the exact expressions of solitary wave solution, singular solitary waves, shock waves, plane waves, and finally singular periodic solutions were obtained. There are also many meaningful results established for fractional Boussinesq equation or generalized Boussinesq systems, readers can refer to [15-20] for details.

However, to the best of our knowledge, some other special solution such as the singular traveling delta wave has not been considered for (1.1) yet. In [21-26], in order to deal with non-smooth or distribution solutions of some nonlinear partial differential equations, such as delta function, Heaviside function etc., the authors have constructed a very suitable definition of products of distribution so that the results remain distributions for any product of distributions. It is a reasonable and effective extension of products of classical functions or distribution multiplied by smooth function, and can return to the classical products if both the factors multiplied by each other are classical functions. We will introduce the details about the products of distributions later. It is worth noticing that, in [21-26], only the first order partial differential models were studied. So far, the higher order partial differential equations, even the second order ones, have not been considered in this way yet. It is a new attempt to use these methods in the above references to study the distribution solutions for a fourth order equation like (1.1). So, in this paper, we use the relative definition and approach on products of distributions therein to research some specific aspects of propagation of delta waves for (1.1). It is proved that, in a sense of the 
products of distributions defined by [21-26], under certain conditions, the traveling delta wave

$$
u(x, t)=m \delta(x-c t)
$$

is a solution of (1.1), where $\delta$ stands for the Dirac measure concentrated at the origin.

First of all, it is necessary to give an overview on the products of distributions, because we have to depend on such products of distributions to obtain the relative results.

Non-smooth functions or singular functions can be regarded as distributions or generalized functions. We have to turn to distributions or generalized functions when we want to obtain non-smooth or singular solutions for nonlinear partial differential equations because of the nonlinearity. Now we recall some results on products of distributions. Firstly, Maslov and his collaborators [27-30] introduced several distribution algebras, and later Rosinger [31-34] did some similar works. These works brought into light algebraic structures involved in embedding the space of distributions $D^{\prime}$ into certain quotient algebras. The article of Egorov [35] is a very good guide for a preliminary review about those types of approaches to products of distributions.

Later, several more products of distributions were introduced, the most popular one is the work of Colombeau [36, 37], it is especially related to the framework of Rosinger. The book of Oberguggenberger [38] can be well referred to in this direction.

As is well known, unfortunately, some distributional products are probably not successful in multiplying distributions with a strong singularity at a given point, for instance, the product $\delta \delta$ of two Dirac-delta measures. Other approaches obtain such products at the price of leaving out the space of distributions. For example, $\delta \delta$ is an element of the Colombeau's algebra G, but this element has no associated distribution. Consequently, from the mathematical point of view, $\delta \delta$ is well defined but difficult to interpret at a level of theoretical physics; some indeterminacies also arise.

The approach in $[21,22]$ is a general theory that provides a distribution as the outcome of any product of distributions, once we fix a certain function $\alpha$. Such a function quantifies the indeterminacy inherent to the products, and, once fixed, its physical interpretation becomes clear. They stress that this indeterminacy is not avoidable in general, and it plays an essential role in many questions. Concerning this point, we can refer to Sect. 6 in [22] and also to [39-41]. For instance, within their framework, they have exhibited explicitly $[21,25]$ Dirac delta wave solutions (and also solutions which are not measures) for the turbulent model ruled by Burgers nonconservative equation, and some phenomena, like "infinitely narrow soliton solutions," obtained by Maslov and his collaborators arise directly in distributional form [25] as a particular case. Also in the same setting, for a model ruled by a singular perturbation of Burgers conservative equation, they have proved [26] that delta-waves under collision behave as classical soliton collisions (as in the Kortewegde Vries equation).

The rest of this paper is organized as follows. In Sect. 2, we give a review about the delta distribution and some of its properties used later. And then we introduce the product of distributions in a particular sense and some arithmetic rules in Sect. 3. In Sect. 4 we define the concept of $\alpha$-solution and show that it is a particular extension of classical solution. Finally, under some conditions, we prove that (1.1) possesses traveling delta wave solutions in Sect. 5. 


\section{Delta function and some of its properties}

In mathematics, the Dirac delta function ( $\delta$ function) is a generalized function or distribution. It is used to model the density of an idealized point mass or point charge as a function equal to zero everywhere except for zero and whose integral over the entire real line is equal to one.

That is,

$$
\delta(x)= \begin{cases}+\infty, & x=0 \\ 0, & x \neq 0\end{cases}
$$

and it is also constrained to satisfy the identity

$$
\int_{-\infty}^{+\infty} \delta(x) d x=1
$$

As there is no function that has these properties, the computations made by the theoretical physicists appeared to mathematicians as nonsense until the introduction of distributions by Laurent Schwartz to formalize and validate the computations. As a distribution, the Dirac delta function is a linear functional that maps every function to its value at zero.

Here we present some properties that will be used later. The delta function satisfies the following scaling property for a nonzero scalar $\mu$ :

$$
\int_{-\infty}^{+\infty} \delta(\mu x) d x=\int_{-\infty}^{+\infty} \delta(y) \frac{d y}{|\mu|}=\frac{1}{|\mu|}
$$

and so

$$
\delta(\alpha x)=\frac{\delta(x)}{|\alpha|} .
$$

In particular, the delta function is an even distribution in the sense that

$$
\delta(-x)=\delta(x)
$$

The distributional derivative of the Dirac delta distribution is the distribution $\delta^{\prime}$ defined on compactly supported smooth test functions $\varphi$

$$
\delta^{\prime}[\varphi]=-\delta\left[\varphi^{\prime}\right]=-\varphi^{\prime}(0) .
$$

The above equality here is a kind of integration by parts, for if $\delta$ were a true function, then

$$
\int_{-\infty}^{\infty} \delta^{\prime}(x) \varphi(x) d x=-\int_{-\infty}^{\infty} \delta(x) \varphi^{\prime}(x) d x
$$

The $k$ th derivative of $\delta$ is defined similarly as the distribution given on test functions by

$$
\delta^{(k)}[\varphi]=(-1)^{k} \varphi^{(k)}(0) .
$$


In particular, $\delta$ is an infinitely differentiable distribution. Furthermore, the convolutions of $\delta$ and $\delta^{\prime}$ with a compactly supported smooth function $f$ are

$$
\delta * f=\delta * f=f
$$

and

$$
\delta^{\prime} * f=\delta * f^{\prime}=f^{\prime}
$$

respectively, which follows from the properties of the distributional derivative of a convolution.

\section{Product of distributions}

This section introduces the product of distributions defined in [21, 22].

Let $\mathcal{D}$ be the space of compactly supported infinitely differentiable complex-valued functions defined on $R$, let $\mathcal{D}^{\prime}$ be the space of Schwartz distributions, and let $\alpha \in \mathcal{D}$ be even with $\int_{-\infty}^{\infty} \alpha=1$. In the theory of products in [21,22], for computing the $\alpha$-product $T_{\dot{\alpha}} S$, they arrive at a relation of the form

$$
T_{\dot{\alpha}} S=T \beta+(T * \alpha) f
$$

for $T \in \mathcal{D}^{\prime}$ and $S=\beta+f \in C^{p} \oplus \mathcal{D}_{\mu}^{\prime}$, where $p \in 0,1,2, \ldots, \infty, \mathcal{D}^{\prime p}$ is the space of distributions of order $p$ in the sense of $\operatorname{Schwartz}\left(\mathcal{D}^{\prime \infty}\right.$ means $\left.\mathcal{D}^{\prime}\right), \mathcal{D}_{\mu}^{\prime}$ is the space of distributions whose support has measure zero in the sense of Lebesgue, and $T \beta$ is the usual Schwartz product of a $\mathcal{D}^{\prime p}$ distribution by a $C^{p}$-function.

Remark 3.1 The $\alpha$-product is a generalization of the classical product of functions in the distribution sense. Therefore, the weak solution of the nonlinear PDE is related to this product. It is clear to see that, in (3.1), if the functions $T$ and $S$ are classical functions, then $S=\beta+f, f=0$ and $T_{\dot{\alpha}} S=T \beta+(T * \alpha) f=T \beta$. Hence the $\alpha$-product is equivalent to the classical product.

For instance, if $\delta$ stands for the Dirac measure, we have

$$
\begin{aligned}
& \delta_{\dot{\alpha}} \delta=\delta_{\dot{\alpha}}(0+\delta)=(\delta * \alpha) \delta=\alpha \delta=\alpha(0) \delta \\
& \delta_{\dot{\alpha}}(D \delta)=(\delta * \alpha)(D \delta)=\alpha(0)(D \delta)-\alpha^{\prime}(0) \delta=\alpha(0)(D \delta), \\
& (D \delta)_{\dot{\alpha}} \delta=((D \delta) * \alpha) \delta=\left(\delta * \alpha^{\prime}\right) \delta=\alpha^{\prime}(0) \delta=0
\end{aligned}
$$

where $D$ denotes the generalized derivative.

It is easy to define the product of a distribution with a smooth function. A limitation of the theory of distributions is that there is no associative product of two distributions extending the product of a distribution by a smooth function, as has been proved by Laurent Schwartz in the 1950s [42, 43]. So about the properties of this kind of product of distributions, it is quite different from the pointwise product of classical functions.

This $\alpha$-product is bilinear, has unit element(the constant function taking the value 1 viewed as a distribution), is invariant under translations and also under the action of the 
transformation $t \rightarrow-t$ from $R$ onto $R$. In general, this product is neither associative nor commutative; however,

$$
\int_{R} T_{\dot{\alpha}} S=\int_{R} S_{\dot{\alpha}} T
$$

for any $\alpha$ if $T, S \in \mathcal{D}_{\mu}^{\prime}$ and $T$ or $S$ is compactly supported. In general, $\alpha$-products cannot be completely localized. This becomes clear by noticing that $\operatorname{supp}\left(T_{\dot{\alpha}} S\right) \subset \operatorname{supp} S$ (as for ordinary functions), but it can happen that $\operatorname{supp}\left(T_{\dot{\alpha}} S\right) \subset \operatorname{supp} T$. Thus, in the following, $\alpha$-product is regarded as a global product, and when we apply the product to differential equations, the solutions are naturally viewed as global solutions. Product (3.1) is consistent with the Schwartz product of $\mathcal{D}^{\prime p}$-distributions by $C^{p}$-functions (if these functions are placed on the right-hand side) and satisfy the standard differential rules.

In general, $\alpha$-product cannot be completely localized. Thus, in the following, $\alpha$-product is regarded as a global product. The Leibniz formula must be represented in the form

$$
D\left(T_{\dot{\alpha}} S\right)=(D T)_{\dot{\alpha}} S+T_{\dot{\alpha}}(D S),
$$

where $D$ is the derivative operator in the distributional sense.

Besides, we can use $\alpha$-products (3.1) to define powers of some distributions. Thus, if $T=\beta+f \in C^{p} \oplus \mathcal{D}_{\mu}^{\prime} \cap \mathcal{D}^{\prime} p$, then

$$
T_{\dot{\alpha}} T=\beta^{2}+[\beta+(\beta * \alpha)+(f * \alpha)] f
$$

because $T \in \mathcal{D}^{\prime} p \cap\left(C^{p} \oplus \mathcal{D}_{\mu}^{\prime}\right)$. Since $T_{\dot{\alpha}} T \in C^{p} \oplus \mathcal{D}_{\mu}^{\prime} \cap \mathcal{D}^{\prime} p$, we can define the $\alpha$-powers $T_{\alpha}^{n}(n \geq 0$ is an integer) by the recurrence formula

$$
\begin{aligned}
& T_{\alpha}^{0}=1, \\
& T_{\alpha}^{n}=\left(T_{\alpha}^{n-1}\right)_{\dot{\alpha}} T .
\end{aligned}
$$

Since the distributional products (3.1) are consistent with the Schwartz products of distributions by functions (when functions are placed on the right-hand side), we have $\beta_{\alpha}^{n}=\beta^{n}$ for all $\beta \in C^{p}$, and the consistency of this definition with the ordinary powers of $C^{p}$ functions is proved. For instance, if $m \in C$, then $(m \delta)_{\alpha}^{0}=1$ and $(m \delta)_{\alpha}^{n}=m^{n}[\alpha(0)]^{n-1}$ for $n \geq 2$, which can readily be seen by induction.

We also have $\left(\tau_{a} T\right)_{\alpha}^{n}=\tau_{a}(T)_{\alpha}^{n}$ in the distributional sense, where $\tau_{a}$ is the translation operator defined by $a \in R$. Thus, in what follows, we shall write $T^{n}$ instead of $T_{\alpha}^{n}$ (supposing that $\alpha$ is fixed), which will also simplify the notation.

Notice that, under the definition of this kind product of distributions, if $\phi(u)$ is an entire function of $u$, then $\phi \circ u$ is well defined, here $\phi \circ u$ is used to denote the expression of $\phi(u)$ involving the product of distributions, and we have the following result.

Lemma 3.1 ([22]) If $\phi(u)$ is an entire function of $u$, then

$$
\phi \circ(m \delta)= \begin{cases}\phi(0)+\phi^{\prime}(0) m \delta & \text { if } \alpha(0)=0, \\ \phi(0)+\frac{\phi[m \alpha(0)]-\phi(0)}{\alpha(0)} \delta & \text { if } \alpha(0) \neq 0 .\end{cases}
$$


Proof If $\phi(u)$ is an entire function of $u$, then we have

$$
\phi(u)=a_{0}+a_{1} u+a_{2} u^{2}+\cdots,
$$

where $a_{n}=\frac{\phi^{n}(0)}{n !}$ for $n=0,1,2, \ldots$ For $T \in C^{p} \oplus\left(\mathcal{D}^{\prime p} \cup \mathcal{D}_{\mu}^{\prime}\right)$, we define the composition $\phi \circ T$ as follows:

$$
\phi \circ T=a_{0}+a_{1} T+a_{2} T^{2}+\cdots
$$

provided this series converges in $\mathcal{D}^{\prime}$. This is clearly a consistent definition, and we have $\tau_{a}(\phi \circ T)=\phi \circ\left(\tau_{a} T\right)$ if $\phi \circ T$ or $\phi \circ\left(\tau_{a} T\right)$ is well defined. Recall that $\phi \circ T$ depends on $\alpha$ in general. Now we shall show that $\phi \circ(m \delta)$ is a distribution for all $m \in \mathbb{C}$. We have $(m \delta)^{0}=1$ and $(m \delta)^{1}=m \delta$ and, for $n \geq 2$,

$$
(m \delta)^{n}=m^{n}[\alpha(0)]^{n-1} \delta
$$

as we have already seen. Then, according to (3.12),

$$
\phi \circ(m \delta)=a_{0}+a_{1} m \delta+a_{2}(m \delta)^{2}+\cdots,
$$

because, as we shall see, this series is convergent in $\mathcal{D}^{\prime}$. Indeed, by (3.13), we have

$$
\phi \circ(m \delta)=a_{0}+a_{1} m \delta+a_{2} m^{2} \alpha(0) \delta+a_{3} m^{3}[\alpha(0)]^{2} \delta+\cdots,
$$

and thus, if $\alpha(0)=0$, then $\phi \circ(m \delta)=a_{0}+a_{1} m \delta$, while if $\alpha(0) \neq 0$, then

$$
\alpha(0)\left[\phi \circ(m \delta)-a_{0}\right]=a_{1} \alpha(0) m \delta+a_{2} m^{2}[\alpha(0)]^{2} \delta+a_{3} m^{3}[\alpha(0)]^{3} \delta+\cdots,
$$

which is equivalent to

$$
\alpha(0)\left[\phi \circ(m \delta)-a_{0}\right]=\left[a_{1} \alpha(0) m+a_{2} m^{2}[\alpha(0)]^{2}+a_{3} m^{3}[\alpha(0)]^{3}+\cdots\right] \delta,
$$

because, by (3.11), the series $\{\cdots\}$ converges to $\phi(m \alpha(0))-a_{0}$. In this case,

$$
\alpha(0)\left[\phi \circ(m \delta)-a_{0}\right]=\left[\phi(m \alpha(0))-a_{0}\right] \delta,
$$

from the above equation, we have

$$
\phi \circ(m \delta)=\phi(0)+\frac{\phi[m \alpha(0)]-\phi(0)}{\alpha(0)} \delta .
$$

This completes the proof.

\section{The concept of $\alpha$-solution}

For simplicity, we first deal with the case $n=1$ for (1.1), that is,

$$
u_{t t}-k^{2} u_{x x}+a\left(u^{2}\right)_{x x}+b_{1} u_{x x x x}+b_{2} u_{x x t t}=0 .
$$

Let us consider equation (4.1). By a classical solution of (4.1) we mean a forth order continuously differentiable complex function $(x, t) \rightarrow u(x, t)$ which satisfies (4.1) at every point 
of its domain. Let $I$ be an interval of $R$ with nonempty interior, and let $F(I)$ be the space of second order continuously differentiable mappings $\tilde{u}: I \rightarrow \mathcal{D}^{\prime}$ in the sense of the topology of $\mathcal{D}^{\prime}$. For $t \in I$, the notation $[\tilde{u}(t)](x)$ is sometimes used to stress that the distribution $\tilde{u}(t)$ acts on functions $\xi \in \mathcal{D}$ that depend on $x$.

Definition 4.1 The mapping $\tilde{u} \in F(I)$ is said to be an $\alpha$-solution of (4.1) if and only if there is an $\alpha$ such that, for all $t \in I$,

$$
\left(1+b_{2} D^{(2)}\right) \frac{d^{2} \widetilde{u}(t)}{d t^{2}}-k^{2} D^{(2)} \widetilde{u}(t)+a D^{(2)}\left(\widetilde{u}(t)_{\dot{\alpha}} \widetilde{u}(t)\right)+b_{1} D^{(4)} \widetilde{u}(t)=0,
$$

where $D^{(n)}(n=2$ or 4$)$ stands for the distributional derivative.

Theorem 4.1 If $u$ is a global classical solution of equation (4.1) on $R \times I$, then, for any $\alpha$, the map $\tilde{u}$ defined by $[\widetilde{u}(t)](x)=u(x, t)$ is a global $\alpha$-solution of $(4.1)$.

Theorem 4.2 If $u: R \times[0,+\infty) \rightarrow C$ is a $C^{4}$-function and $\tilde{u}:[0,+\infty) \rightarrow D^{\prime}$ defined by $[\widetilde{u}(t)](x)=u(x, t)$ is a global $\alpha$-solution of (4.1), then $u$ is a global classical solution of (4.1).

For the proof, it is sufficient to note that a $C^{4}$-function $u(x, t)$ can be treated as a continuously differentiable function $\widetilde{u} \in F(I)$ defined by $[\widetilde{u}(t)](x)=u(x, t)$ and to use the consistency of the $\alpha$-products with the classical ones.

Remark 4.1 Theorems 4.1 and 4.2 show that $\alpha$-solution is a particular extension of the classical solution.

\section{The propagation of a wave profile $T \in \mathcal{D}^{\prime}$}

Definition 5.1 Let $\tau_{c t}$ be a translation operator satisfying $\tau_{c t} T(\cdot)=T(\cdot-c t)$. We say that $T \in D^{\prime} \alpha$-propagates with the speed $c$, according to (4.1), if and only if the mapping $\tilde{u} \in F(I)$ defined by $\widetilde{u}(t)=\tau_{c t} T$ is an $\alpha$-solution of (4.1).

Theorem 5.1 Let $T \in D^{\prime}$ be a nonconstant distribution. Then $T \alpha$-propagates with the speed c, according to (4.1), if and only if

$$
c^{2}\left(1+b_{2} D^{(2)}\right) D^{(2)} T-k^{2} D^{(2)} T+a D^{(2)}\left(T_{\dot{\alpha}} T\right)+b_{1} D^{(4)} T=0 .
$$

Proof Assume that $T \alpha$-propagates with the speed $c$. By Definitions 4.1 and 5.1 we have

$$
\left(1+b_{2} D^{(2)}\right) \frac{d^{2}\left(\tau_{c t} T\right)}{d t^{2}}-k^{2} D^{(2)}\left(\tau_{c t} T\right)+a D^{(2)}\left(\left(\tau_{c t} T\right)_{\dot{\alpha}}\left(\tau_{c t} T\right)\right)+b_{1} D^{(4)}\left(\tau_{c t} T\right)=0
$$

for all $t \in I$.

According to [13, p. 648], we have $\frac{d^{2}\left(\tau_{c t} T\right)}{d t^{2}}=c^{2} D^{(2)}\left(\tau_{c t} T\right)$, so the above equation can be rewritten as

$$
c^{2}\left(1+b_{2} D^{(2)}\right) D^{(2)}\left(\tau_{c t} T\right)-k^{2} D^{(2)}\left(\tau_{c t} T\right)+a D^{(2)}\left(\left(\tau_{c t} T\right)_{\dot{\alpha}}\left(\tau_{c t} T\right)\right)+b_{1} D^{(4)}\left(\tau_{c t} T\right)=0 .
$$

Using the translation operator $\tau_{-c t}$ to the above equation, we have

$$
c^{2}\left(1+b_{2} D^{(2)}\right) D^{(2)} T-k^{2} D^{(2)} T+a D^{(2)}\left[\tau_{-c t}\left(\left(\tau_{c t} T\right)_{\dot{\alpha}}\left(\tau_{c t} T\right)\right)\right]+b_{1} D^{(4)} T=0 .
$$


Again, since $\tau_{c t}\left(T^{2}\right)_{\dot{\alpha}}=\left(\tau_{c t} T\right)_{\dot{\alpha}}^{2}$, it follows that

$$
c^{2}\left(1+b_{2} D^{(2)}\right) D^{(2)} T-k^{2} D^{(2)} T+a D^{(2)}\left(T_{\dot{\alpha}} T\right)+b_{1} D^{(4)} T=0 .
$$

Especially, if $T=m \delta(x)$, it can be verified that

$$
\frac{d^{2}\left(\tau_{c t} m \delta(x)\right)}{d t^{2}}=c^{2} D^{(2)}\left(\tau_{c t} m \delta(x)\right) .
$$

Let $\xi \in \mathcal{D}$ be a test function, in fact,

$$
\begin{aligned}
\left\langle\frac{d\left(\tau_{c t} m \delta(x)\right)}{d t}, \xi\right\rangle & =\left\langle\frac{d(m \delta(x-c t))}{d t}, \xi\right\rangle \\
& =\left\langle\lim _{h \rightarrow 0} \frac{m \delta(x-c(t+h))-m \delta(x-c t)}{h}, \xi\right\rangle \\
& =\lim _{h \rightarrow 0} \frac{1}{h}[\langle m \delta(x-c(t+h)), \xi\rangle-\langle m \delta(x-c t), \xi\rangle] \\
& =\lim _{h \rightarrow 0} \frac{1}{h}[m \xi(c(t+h))-m \xi(c t)] \\
& =c m \xi^{\prime}(c t) \\
& =c m\left\langle\delta(x-c t), \xi^{\prime}(x)\right\rangle \\
& =-c m\left\langle\delta^{\prime}(x-c t), \xi(x)\right\rangle \\
& =\left\langle-c m \delta^{\prime}(x-c t), \xi(x)\right\rangle,
\end{aligned}
$$

that is,

$$
\frac{d\left(\tau_{c t} m \delta(x)\right)}{d t}=-m c \delta^{\prime}(x-c t)
$$

where the prime of $\delta$ is the distributional derivative. Similarly, we have

$$
\begin{aligned}
\left\langle\frac{d^{2}\left(\tau_{c t} m \delta(x)\right)}{d t^{2}}, \xi\right\rangle & =\left\langle\frac{d^{2}(m \delta(x-c t))}{d t^{2}}, \xi\right\rangle \\
& =\left\langle\lim _{h \rightarrow 0} \frac{c m \delta^{\prime}(x-c(t+h))-c m \delta^{\prime}(x-c t)}{h}, \xi\right\rangle \\
& =\lim _{h \rightarrow 0} \frac{1}{h}\left[\left\langle c m \delta^{\prime}(x-c(t+h)), \xi\right\rangle-\left\langle c m \delta^{\prime}(x-c t), \xi\right\rangle\right] \\
& =\lim _{h \rightarrow 0} \frac{1}{h}\left[c m \xi^{\prime}(c(t+h))-c m \xi^{\prime}(c t)\right] \\
& =c^{2} m \xi^{\prime \prime}(c t) \\
& =c^{2} m\left\langle\delta(x-c t), \xi^{\prime \prime}(x)\right\rangle \\
& =-c^{2} m\left\langle\delta^{\prime}(x-c t), \xi^{\prime}(x)\right\rangle \\
& =\left\langle c^{2} m \delta^{\prime \prime}(x-c t), \xi(x)\right\rangle
\end{aligned}
$$


namely,

$$
\frac{d^{2}\left(\tau_{c t} m \delta(x)\right)}{d t^{2}}=c^{2} D^{(2)}(m \delta(x-c t))=c^{2} D^{(2)}\left(\tau_{c t} m \delta(x)\right) .
$$

Now we show that a Dirac delta wave $T=m \delta \alpha$-propagating with speed $c$ is a solution of (4.1), $m \in C$ is a nonzero constant.

Theorem 5.2 Dirac delta wave $T=m \delta \alpha$-propagates with speed c according to (4.1) if and only if one of the following two conditions is satisfied:

(1) If $\alpha(0)=0$, then $c^{2}=k^{2}=-\frac{b_{1}}{b_{2}}$.

(2) If $\alpha(0) \neq 0$, the wave speed c satisfies $c^{2}=-\frac{b_{1}}{b_{2}}$, the $\alpha$ function should be chosen with $\alpha(0)=\frac{b_{1}+k^{2} b_{2}}{a b_{2} m}$.

Proof According to the definition of product of distributions and Lemma 3.1, calculating directly, we have

$$
\phi \circ(m \delta)=m \delta(x)_{\dot{\alpha}} m \delta(x)= \begin{cases}0 & \text { if } \alpha(0)=0, \\ m^{2} \alpha(0) \delta(x) & \text { if } \alpha(0) \neq 0 .\end{cases}
$$

By using Theorem 5.1, substituting (5.25) into (5.1), we have

$$
c^{2}\left(1+b_{2} D^{(2)}\right) m D^{(2)} \delta(x)-k^{2} m D^{(2)} \delta(x)+a D^{(2)}\left(m^{2} \alpha(0) \delta(x)\right)+b_{1} m D^{(4)} \delta(x)=0,
$$

or

$$
c^{2}\left(1+b_{2} D^{(2)}\right) m D^{(2)} \delta(x)-k^{2} m D^{(2)} \delta(x)+b_{1} m D^{(4)} \delta(x)=0,
$$

that is,

$$
\left[c^{2}-k^{2}+a m \alpha(0)\right] D^{(2)} \delta(x)+\left(c^{2} b_{2}+b_{1}\right) D^{(4)} \delta(x)=0,
$$

or

$$
\left(c^{2}-k^{2}\right) D^{(2)} \delta(x)+\left(c^{2} b_{2}+b_{1}\right) D^{(4)} \delta(x)=0,
$$

the above equation holds true if and only if $c^{2}=-\frac{b_{1}}{b_{2}}$ and $\alpha(0)=\frac{b_{1}+k^{2} b_{2}}{a b_{2} m}$ or $c^{2}=k^{2}=-\frac{b_{1}}{b_{2}}$. When $n=2$, equation (1.1) becomes

$$
u_{t t}-k^{2} u_{x x}+a\left(u^{4}\right)_{x x}+b_{1} u_{x x x x}+b_{2} u_{x x t t}=0
$$

Following the above steps, we can obtain the $\delta$ solution for (5.30) by the following theorem.

Theorem 5.3 Dirac delta wave $T=m \delta \alpha$-propagates with speed $c$ according to (5.30) if and only if one of the following two conditions is satisfied: 
(1) If $\alpha(0)=0$, then $c^{2}=k^{2}=-\frac{b_{1}}{b_{2}}$.

(2) If $\alpha(0) \neq 0$, the wave speed c satisfies $c^{2}=-\frac{b_{1}}{b_{2}}$, the $\alpha$ function should be chosen with $\alpha(0)=\frac{1}{m} \sqrt[3]{\frac{b_{1}+k^{2} b_{2}}{a b_{2}}}$.

Proof On the basis of the definition of product of distributions and Lemma 3.1, calculating directly, we have

$$
\phi \circ(m \delta)=[m \delta(x)]_{\dot{\alpha}}^{4}= \begin{cases}0 & \text { if } \alpha(0)=0, \\ m^{4}[\alpha(0)]^{3} \delta(x) & \text { if } \alpha(0) \neq 0,\end{cases}
$$

where $[m \delta(x)]_{\dot{\alpha}}^{4}=m \delta(x)_{\dot{\alpha}} m \delta(x)_{\dot{\alpha}} m \delta(x)_{\dot{\alpha}} m \delta(x)$. Then, substituting (5.31) into the following equation

$$
c^{2}\left(1+b_{2} D^{(2)}\right) D^{(2)} T-k^{2} D^{(2)} T+a D^{(2)}\left(T_{\dot{\alpha}}^{4}\right)+b_{1} D^{(4)} T=0,
$$

where $T_{\dot{\alpha}}^{4}=T_{\dot{\alpha}} T_{\dot{\alpha}} T_{\dot{\alpha}} T$, we have

$$
c^{2}\left(1+b_{2} D^{(2)}\right) m D^{(2)} \delta(x)-k^{2} m D^{(2)} \delta(x)+a D^{(2)}\left(m^{4}[\alpha(0)]^{3} \delta(x)\right)+b_{1} m D^{(4)} \delta(x)=0,
$$

or

$$
c^{2}\left(1+b_{2} D^{(2)}\right) m D^{(2)} \delta(x)-k^{2} m D^{(2)} \delta(x)+b_{1} m D^{(4)} \delta(x)=0,
$$

that is,

$$
\left(c^{2}-k^{2}+a[m \alpha(0)]^{3}\right) D^{(2)} \delta(x)+\left(c^{2} b_{2}+b_{1}\right) D^{(4)} \delta(x)=0,
$$

or

$$
\left(c^{2}-k^{2}\right) D^{(2)} \delta(x)+\left(c^{2} b_{2}+b_{1}\right) D^{(4)} \delta(x)=0,
$$

the above equation holds true if and only if $c^{2}=-\frac{b_{1}}{b_{2}}$ and $\alpha(0)=\frac{1}{m} \sqrt[3]{\frac{b_{1}+k^{2} b_{2}}{a b_{2}}}$ or $c^{2}=k^{2}=$ $-\frac{b_{1}}{b_{2}}$.

Furthermore, with basically similar steps, we can get the traveling delta wave solution of (1.1) for any positive integer $n$.

Theorem 5.4 Dirac delta wave $T=m \delta \alpha$-propagates with speed c according to (1.1) if and only if the following two conditions are satisfied:

(1) If $\alpha(0)=0$, then $c^{2}=k^{2}=-\frac{b_{1}}{b_{2}}$.

(2) If $\alpha(0) \neq 0$, the wave speed $c$ satisfies $c^{2}=-\frac{b_{1}}{b_{2}}$, the $\alpha$ function should be chosen with

$$
\alpha(0)=\frac{1}{m} \sqrt[2 n-1]{\frac{b_{1}+k^{2} b_{2}}{a b_{2}}} .
$$




\section{Conclusion}

Up to now, only first and second order nonlinear partial differential equations have been investigated with this kind of product of distributions. This paper has extended the application of such product of distributions into a higher order nonlinear partial differential equation; under some conditions, it is verified that Dirac delta function with a translation at speed $c$ is a singular solution of (1.1). The result of this paper shows that more higher order nonlinear models are able to be dealt with in this way.

Acknowledgements

The authors would like to thank the reviewers for helpful comments and editors for their hard work.

Funding

This work was supported by the Natural Science Foundation of China (11771151).

Availability of data and materials

Not applicable.

Competing interests

The authors declare that they have no competing interests.

Authors' contributions

The authors contributed equally to this paper. All authors read and approved the final manuscript.

\section{Author details}

'Department of Basic Courses, Guangzhou Maritime University, Guangzhou, P.R. China. ²Department of Mathematics,

Foshan University, Foshan, P.R. China. ${ }^{3}$ Department of Mathematics, Guizhou University, Guiyang, P.R. China.

\section{Publisher's Note}

Springer Nature remains neutral with regard to jurisdictional claims in published maps and institutional affiliations.

Received: 1 May 2019 Accepted: 21 November 2019 Published online: 09 December 2019

\section{References}

1. Boussinesq, J.: Theory of wave and swells propagated in long horizontal rectangular canal and imparting to the liquid contained in this canal. J. Math. Pures Appl. 17(2), 55-108 (1872)

2. Zhang, X., Shu, T., Cao, H., et al.: The general solution for impulsive differential equations with Hadamard fractional derivative of order $q \in(1,2)$. Adv. Differ. Equ. 2016(1), Article ID 14 (2016)

3. Agarwal, P., Dragomir, S.S., Jeeli, M., Samet, B.: Advances in Mathematical Inequalities and Applications. Trends in Mathematics (2019)

4. Tariboon, J., Ntouyas, S.K., Sutthasin, B.: Impulsive fractional quantum Hahn difference boundary value problems. Adv. Differ. Equ. 2019(1), Article ID 220 (2019)

5. Sitho, S., Ntouyas, S.K., Agarwal, P., et al.: Noninstantaneous impulsive inequalities via conformable fractional calculus. J. Inequal. Appl. 2018(1), Article ID 261 (2018)

6. Ruzhansky, M., Je, C.Y., Agarwal, P.: Advances in Real and Complex Analysis with Applications. Trends in Mathematics (2017)

7. Agarwal, P., Ibrahim, I.H., Yousry, F.M.: G-stability one-leg hybrid methods for solving DAEs. Adv. Differ. Equ. 2019(1), Article ID 103 (2019)

8. Saoudi, K., Agarwal, P., Kumam, P., et al.: The Nehari manifold for a boundary value problem involving Riemann-Liouville fractional derivative. Adv. Differ. Equ. 2018(1), Article ID 263 (2018)

9. Hammouch, Z., Mekkaoui, T., Agarwal, P.: Optical solitons for the Calogero-Bogoyavlenskii-Schiff equation in $(2+1)$ dimensions with time-fractional conformable derivative. Eur. Phys. J. Plus 133(7), Article ID 248 (2018)

10. Saad, K., Iyiola, S., Agarwal, P.: An effective homotopy analysis method to solve the cubic isothermal auto-catalytic chemical system. AlMS Math. 3(1), 183-194 (2018)

11. Biswas, A., Song, M., Triki, H., Kara, A.H., Ahmed, B.S., Strong, A., Hama, A.: Solitons, shock waves, conservation laws and bifurcation analysis of Boussinesq equation with power law nonlinearity and dual dispersion. Appl. Math. Inf. Sci. 8, 949-957 (2014)

12. Biswas, A., Milovic, D., Ranasinghe, A.: Solitary waves of Boussinesq equation in a power law media. Commun. Nonlinear Sci. Numer. Simul. 14, 3738-3742 (2009)

13. Ekici, M., Mirzazadeh, M., Eslami, M.: Solitons and other solutions to Boussinesq equation with power law nonlinearity and dual dispersion. Nonlinear Dyn. 84, 669-676 (2016)

14. Polat, N., Piskin, E.: Existence and asymptotic behavior of solution of the Cauchy problem for the damped sixth-order Boussinesq equation. Acta Math. Appl. Sin. Engl. Ser. 31, 735-746 (2015)

15. Baleanu, D., Inc, M., Aliyu, A.I., Yusuf, A.: The investigation of soliton solutions and conservation laws to the coupled generalized Schrödinger-Boussinesq system. Waves Random Complex Media 29(1), 77-92 (2018)

16. Tchier, F., Yusuf, A., Aliyu, A.I., Baleanu, D.: Time fractional third-order variant Boussinesq system: symmetry analysis, explicit solutions, conservation laws and numerical approximations. Eur. Phys. J. Plus 133(6), Article ID 240 (2018) 
17. Javeed, S., Saif, S., Waheed, A., Baleanu, D.: Exact solutions of fractional mBBM equation and coupled system of fractional Boussinesq-Burgers. Results Phys. 9, 1275-1281 (2018)

18. Osman, M.S., Machado, J.A.T., Baleanu, D.: On nonautonomous complex wave solutions described by the coupled Schrödinger-Boussinesq equation with variable-coefficients. Opt. Quantum Electron. 50(2), Article ID 73 (2018)

19. Yang, X.J., Machado, J.A.T., Baleanu, D.: Exact traveling-wave solution for local fractional Boussinesq equation in fractal domain. Fractals 25(4), Article ID 1740006 (2017)

20. Kumar, S., Kumar, A., Baleanu, D.: Two analytical methods for time-fractional nonlinear coupled Boussinesq-Burger's equations arise in propagation of shallow water waves. Nonlinear Dyn. 85(2), 699-715 (2016)

21. Sarrico, C.O.R.: Distributional products and global solutions for nonconservative inviscid Burgers equation. J. Math. Anal. Appl. 281, 641-656 (2003)

22. Sarrico, C.O.R.: Products of distributions and singular travelling waves as solutions of advection-reaction equations. Russ. J. Math. Phys. 19, 244-255 (2012)

23. Sarrico, C.O.R.: About a family of distributional products important in the applications. Port. Math. 45, 295-316 (1988)

24. Sarrico, C.O.R.: Distributional products with invariance for the action of unimodular groups. Riv. Mat. Univ. Parma 4, 79-99 (1995)

25. Sarrico, C.O.R.: New solutions for the one-dimensional nonconservative inviscid Burgers equation. J. Math. Anal. Appl. 317, 496-509 (2006)

26. Sarrico, C.O.R.: Collision of delta-waves in a turbulent model studied via a distribution product. Nonlinear Anal. 73, 2868-2875 (2010)

27. Danilov, V.G., Maslov, V.P., Shelkovich, V.M.: Algebras of singularities of singular solutions to first-order quasi-linear strictly hyperbolic systems. Teor. Mat. Fiz. 114(1), 3-55 (1998). English translation: Theor. Math. Phys. 114(1), 1-42 (1998)

28. Danilov, V.G., Shelkovich, V.M.: Generalized solutions of nonlinear differential equations and the Maslov algebras of distributions. Integral Transforms Spec. Funct. 6(1-4), 171-180 (1998)

29. Maslov, V.P.: Nonstandard characteristics in asymptotical problems. Usp. Mat. Nauk 38(6), 3-36 (1983). English translation: Russ. Math. Surv. 38(6), 1-42 (1983)

30. Maslov, V.P., Tsupin, V.A.: Necessary conditions for existence of infinitely narrow solitons in gas dynamics. Dokl. Akad. Nauk SSSR 246(2), 298-300 (1979). English translation: Sov. Phys. Dokl. 24(5), 354-356 (1979)

31. Rosinger, E.E.: Distributions and Nonlinear Partial Differential Equations. Lecture Notes Math., vol. 684. Springer, Berlin (1978)

32. Rosinger, E.E.: Nonlinear Partial Differential Equations. Sequential and Week Solutions. North Holland, Amsterdam (1980)

33. Rosinger, E.E.: Generalized Solutions of Nonlinear Partial Differential Equations. North Holland, Amsterdam (1987)

34. Rosinger, E.E.: Nonlinear Partial Differential Equations. An Algebraic View of Generalized Solutions. North Holland, Amsterdam (1990)

35. Egorov, Y.V.: On the theory of generalized functions. Usp. Mat. Nauk 45(5), 3-40 (1990). English translation: Russ. Math Surv. 45(5), 1-49 (1990)

36. Colombeau, J.F.: New Generalized Functions and Multiplication of Distributions. North-Holland, Amsterdam (1985)

37. Colombeau, J.F.: Elementary Introduction to New Generalized Functions. North Holland, Amsterdam (1985)

38. Oberguggenberger, M.: Multiplication of Distributions and Applications to Partial Differential Equations. Longman, Harlow (1992)

39. Bressan, A., Rampazzo, F.: On differential systems with vector-valued impulsive controls. Boll. Unione Mat. Ital. 2B(7), 641-656 (1988)

40. Colombeau, J.F., Roux, A.L.: Multiplication of distributions in elasticity and hydrodynamics. J. Math. Phys. 29, 315-319 (1988)

41. Maso, G.D., LeFloch, P., Murat, F.: Definitions and weak stability of nonconservative products. J. Math. Pures Appl. 74 483-548 (1995)

42. Schwartz, L.: Théorie des Distributions, vol. I (1950)

43. Schwartz, L.: Théorie des Distributions, vol. II (1951)

\section{Submit your manuscript to a SpringerOpen ${ }^{\circ}$ journal and benefit from:}

- Convenient online submission

- Rigorous peer review

- Open access: articles freely available online

- High visibility within the field

- Retaining the copyright to your article

Submit your next manuscript at $>$ springeropen.com 